\title{
Adults' number-line estimation strategies: Evidence from eye movements
}

\author{
Jessica L. Sullivan • Barbara J. Juhasz • \\ Timothy J. Slattery $\cdot$ Hilary C. Barth
}

Published online: 16 March 2011

(C) The Author(s) 2011. This article is published with open access at Springerlink.com

\begin{abstract}
Although the development of number-line estimation ability is well documented, little is known of the processes underlying successful estimators' mappings of numerical information onto spatial representations during these tasks. We tracked adults' eye movements during a number-line estimation task to investigate the processes underlying number-to-space translation, with three main results. First, eye movements were strongly related to the target number's location, and early processing measures directly predicted later estimation performance. Second, fixations and estimates were influenced by the size of the first number presented, indicating that adults calibrate their estimates online. Third, adults' number-line estimates demonstrated patterns of error consistent with the predictions of psychophysical models of proportion estimation, and eye movement data predicted the specific error patterns we observed. These results support proportion-based accounts of number-line estimation and suggest that adults' translation of numerical information into spatial representations is a rapid, online process.
\end{abstract}

Keywords Mathematical cognition - Eye movements .

Visual attention

The nature of our ability to relate numerical and spatial representations (e.g., de Hevia \& Spelke, 2010; Hubbard, Piazza, Pinel, \& Dehaene, 2005) is of great current interest,

J. L. Sullivan · B. J. Juhasz $\cdot$ H. C. Barth

Department of Psychology, Wesleyan University,

Middletown, CT 06459, USA

J. L. Sullivan $(\bowtie) \cdot$ T. J. Slattery

Department of Psychology, University of California at San Diego,

9500 Gilman \#0109,

La Jolla, CA 92093, USA

e-mail: jsulliva@ucsd.edu in part because of its relation to children's math achievement (e.g., Siegler \& Ramani, 2009; Siegler, Thompson, \& Opfer, 2009). Investigators commonly explore this ability with number-line estimation tasks, in which participants indicate where a number belongs on a line spanning some numerical range (e.g., Booth \& Siegler, 2006; Siegler \& Opfer, 2003). Number-line estimation requires translating external representations of number (often numerals) into external spatial representations (positions on a line). These tasks involve at least three number-related components: observers' understanding of symbolic number systems, mental representations of numerical quantity, and strategies for mapping numerical information onto space.

What we learn about mental representations of number from these tasks is disputed (e.g., Barth \& Paladino, 2011; Cantlon, Cordes, Libertus, \& Brannon, 2009; Cohen \& Blanc-Goldhammer, in press; Dehaene, Izard, Pica, \& Spelke, 2009; Ebersbach, Luwel, Frick, Onghena, \& Verschaffel, 2008). For example, Siegler and colleagues have found that relatively younger children produce logarithmic-appearing error patterns in these tasks, whereas relatively older children and adults produce more linearappearing estimates (e.g., Siegler \& Opfer, 2003; Siegler et al., 2009). They interpreted these data as evidence for a shift from logarithmic to linear mental representations of number. Other researchers have argued that developmental changes in estimation performance may implicate different kinds of changes in mental number representations. For example, Barth and Paladino (2011) argued that numberline tasks should be treated as proportion estimation tasks (see Cohen, Ferrell, \& Johnson, 2002; Hollands \& Dyre, 2000, for reviews) because number-line estimation tasks entail part/whole judgments of numerical magnitude.

Formal models of proportion estimation have been developed in the context of perceptual magnitude judg- 
ments; in these models, estimates of proportions result from biased estimates of part and whole magnitudes, leading to characteristic S-shaped or reverse S-shaped curves (Hollands \& Dyre, 2000; Hollands, Tanaka, \& Dyre, 2002; Spence, 1990). These models have been found to account for performance in a variety of implicit and explicit perceptual proportion tasks (Hollands \& Dyre, 2000). Barth and Paladino (2011) applied proportionalreasoning models to children's number-line estimations. These models made accurate quantitative predictions about children's estimation biases without appealing to a shift from logarithmic to linear representations, suggesting instead that mental representations of number may undergo smooth developmental change. Greater estimation accuracy, on this view, also results from the use of reference points (e.g., from children's knowledge that 50 should be located in the middle of a $0-100$ number line), and the use of such reference points leads to explicit quantitative predictions as well (e.g., Hollands \& Dyre, 2000). Cohen and BlancGoldhammer (in press) extended proportion estimation models to adults' number-line estimation, finding support for these models in adults and providing suggestive evidence of developmental continuity in mental representations of number. ${ }^{1}$

We also know relatively little about the role of participants' strategies in number-line estimation tasks. Previous studies using different estimation methodologies suggested that inferences about the range of numbers tested may influence performance: Observers adjust their verbal estimates of the number of items in sets online, in response to information about the size and range of to-be-estimated numbers (Izard \& Dehaene, 2008; Sullivan \& Barner, 2010). These findings suggest that adults can strategically adjust word-to-number mappings in response to task demands, but the possibility of online calibration of estimation behavior during number-line estimation has not been explored.

Here, we examine adults' mappings between numbers and positions by recording eye movements during a number-line estimation task. There is strong evidence that eye movements can provide insight into how adults and children process numerical information (e.g., Heine et al., 2010; Loetscher, Bockisch, Nicholls, \& Brugger, 2010; Schneider et al., 2008; Schwarz \& Keus, 2004), and because adults are extremely accurate at number-line estimation (e.g., Siegler \& Opfer, 2003), eye movement measures may be especially informative about their estimation behavior.

\footnotetext{
${ }^{1}$ Although Siegler and Opfer (2003) proposed a "landmark-based proportionality" strategy for number-line estimation, their proposal was based on analyses both methodologically and theoretically unrelated to the formal models developed by Spence (1990) and Hollands and Dyre (2000).
}

Two previous studies have related eye movements to performance in a $0-100$ number-line task in children (Heine et al., 2010; Schneider et al., 2008). Schneider et al. demonstrated that eye movement accuracy was related to estimation accuracy, that estimation ability improved with age, and that children fixated on the endpoints and midpoint of the line. Heine et al. also found a relationship between eye movements and estimation. However, in these studies, accuracy was measured coarsely (with $20 \%$ of the number line considered correct; Schneider et al., 2008); data from early fixations were excluded; eye-tracking analyses were conducted during correct/incorrect judgments, not during estimation (Heine et al., 2010); and visual stimulus placement may have biased looking behavior.

Our study had three major aims. First, we sought to characterize the relationship between early task processing and eventual estimation. To do so, we examined the time course over which participants oriented themselves to the correct estimation location, as well as the relationship between fixation locations and estimation error. Second, we asked whether adults calibrate estimates online, by testing the influence of task demands on number-to-space translation, since this possibility has not been investigated previously in number-line estimation. In pursuit of this aim, we varied the numerals to be estimated and the magnitude of the first numeral encountered. If adults rely largely on memorized correspondences between particular numbers and locations, these manipulations should not influence their estimates. However, if participants calibrate estimates online, the manipulations might influence later estimation. Third, we determined whether adults' numberline estimations and fixation patterns showed that they treated the task as a proportion estimation problem, as has been suggested by previous studies in children (Barth \& Paladino, 2011) and adults (Cohen \& Blanc-Goldhammer, in press). To do so, we examined the amount and direction of estimation error to find out whether small deviations from adults' characteristic near-linear performance, though not predicted by the logarithmic-to-linear-shift hypothesis, conformed to the predictions of the proportional-reasoning account. We then asked whether fixation data supported the same account. This experiment is therefore the first to investigate whether eye movements and estimation data can provide converging evidence of proportion estimation accounts.

\section{Method}

Participants

A total of 22 adults with normal or corrected-to-normal vision participated in exchange for a prize. Of these 
participants, 3 were excluded for substantial track loss, 1 for failure to complete all trials, and 2 for inaccurate eyetracking calibrations. Thus, 16 participants were included in the final analyses.

\section{Materials}

The number lines were horizontal black lines 830 pixels long, with endpoints marked " 0 " and " 1000 ," centered on a gray background. The numbers to be estimated were aurally presented to avoid influencing eye fixations.

\section{Apparatus}

An EyeLink 1000 (SR Research, Ltd) eyetracker recorded eye movements from the right eye. Participants sat $83 \mathrm{~cm}$ from the display (20-in. ViewSonic CRT monitor), with head movements minimized by head-and-chin rests. The stimuli were viewed binocularly.

\section{Procedure}

Eye movements were calibrated with a 9-point full-screen calibration before the experiment and after every five trials. Participants closed their eyes, heard a spoken number word, looked at a gaze-contingent box in the screen's upper left corner to reveal the number line, and produced estimates by mouse-clicking on the line. Eye movements were recorded from the number line's appearance to the participant's estimation via mouse click. The cursor was visible on the screen throughout the trial.

Half of the participants were in the small-initial-number first condition. They estimated 20 randomly selected and pseudorandomly ordered numbers between 1 and 1,000, with the first number presented being relatively small (240). The other half were in the large-initial-number first condition, where they estimated numbers generated by subtracting the small-first numbers from 1,000 , with the first number presented being larger (760). In both initial-number conditions, about half (either $9 / 20$ or $11 / 20$ ) of the requested numbers were $>500$.

\section{Data analysis}

Eye position and mouse clicks were processed using a custom Perl program, which removed blinks and track losses and located each fixation within particular regions of interest (ROIs) for initial data analysis. Each region was 40 pixels wide (4.8\% of the line) and 400 pixels tall. For analyses involving click location, we excluded 4/320 trials due to error exceeding 200 pixels. Additional data analysis was conducted on fixations spanning the entire number line using linear mixed models (LMMs).

\section{Results}

Relation of eye movements to target number

For each trial, the "correct" region was centered on the number's correct location. The 40-pixel regions immediately to the left ("underestimated") and right ("overestimated") of the correct region were also examined. For any trial, the 120 pixels in these three regions comprised less than $15 \%$ of the number line, but contained approximately $50 \%$ of fixations. Although previous studies have used larger ROIs in their analyses of eye movement data (e.g., Heine et al., 2010; Schneider et al., 2008), these larger ROIs prevented the analysis of subtle patterns of over- and underestimation.

Total fixation duration and total number of fixations in these regions were analyzed, indicating the total processing resources devoted to each region during a trial. If a region was not fixated during a trial, a zero was averaged into its mean. The data were analyzed via 2 (initial number: small or large) by 3 (region: correct, underestimate, or overestimate) repeated measures ANOVAs. Error variance was computed over trials. Initial number was a between-trials variable, and region was a within-trials variable (means are displayed in Table 1).

The total number and duration of fixations differed significantly as a function of region [duration, $F(2,76)=$ 13.60, $M S E=171,966, p<.001$; \# fixations, $F(2,76)=$ 11.46, $M S E=0.335, p<.001]$, demonstrating that participants' allocation of processing resources differed in response to the target location. There was a main effect of initial number [duration, $F(1,38)=5.33, M S E=127,282, p<$ .05 ; \# fixations, $F(1,38)=16.05, M S E=0.260, p<.001]$, and initial number interacted with region [duration, $F(2,76)=$ 4.33, $M S E=171,966, p<.025$; \# fixations, $F(2,76)=7.00$, $M S E=0.335, p<.01]$, indicating that looking behavior was influenced by the order of the numbers presented.

Further $t$-tests confirmed that the small-initial-number participants had longer total fixation durations and more fixations overall in the correct region than in either the underestimated or the overestimated region (all $p \mathrm{~s}<.025$ ). These participants also made more fixations in the overestimated region than in the underestimated region $(p<.05)$. The large-initial-number participants had longer total fixation durations and more fixations overall in the correct region than in the overestimated region $(p \mathrm{~s}<.01)$, although total fixation duration and total number of fixations in the underestimated region did not differ from the values for the correct region $(p>.1)$. Additionally, these participants made marginally more fixations in the underestimated than in the overestimated region, $p=.081$, a pattern that is the reverse of the one seen with the smallinitial-number participants. 
Table 1 Means of total fixation durations (in milliseconds) and total numbers of fixations (standard deviations in parentheses)

\begin{tabular}{|c|c|c|c|c|c|c|}
\hline \multirow[t]{2}{*}{ Measures } & \multicolumn{3}{|c|}{ Small initial number } & \multicolumn{3}{|c|}{ Large initial number } \\
\hline & $\begin{array}{l}\text { Correct } \\
\text { region }\end{array}$ & $\begin{array}{l}\text { Overestimate } \\
\text { region }\end{array}$ & $\begin{array}{l}\text { Underestimate } \\
\text { region }\end{array}$ & $\begin{array}{l}\text { Correct } \\
\text { region }\end{array}$ & $\begin{array}{l}\text { Overestimate } \\
\text { region }\end{array}$ & $\begin{array}{l}\text { Underestimate } \\
\text { region }\end{array}$ \\
\hline Total fixation duration & $1,028(461)$ & $641(366)$ & $392(355)$ & $754(529)$ & $301(214)$ & $554(380)$ \\
\hline Total number of fixations & $1.73(0.68)$ & $1.26(0.61)$ & $0.73(0.56)$ & $1.08(0.54)$ & $0.60(0.40)$ & $0.91(0.52)$ \\
\hline
\end{tabular}

Relation of eye movements to estimates

We explored whether the bias in fixation behavior induced by our initial-number manipulation also appeared in estimation behavior. LMMs were used for these analyses, using the Lme4 mixed-effects modeling package in R (Bates \& Sarkar, 2007; R Development Core Team, 2010). These analyses took into consideration all fixations on the number line, not just those in the ROIs analyzed above. All factors were considered fixed effects, except for the random effect of participant. We report the linear slope coefficient $(\beta)$, standard error estimates, and $p$ values estimated from Markov chain Monte Carlo simulations (see Baayen, 2008). A model predicting estimates from target number and initial number indicated a close relationship between target number and the eventual estimate $(\beta=.99, S E=.012, p=.0001$; perfectly linear performance is $\beta=1$ ). However, consistent with the eye movement data presented above, we also found an effect of initial number, such that small-initial-number participants, on average, provided larger estimates than did large-initialnumber participants $(\beta=-23.8, S E=11.7, p<.05)$. There was no interaction $(p>.5)$.

Next, we assessed the relationship between first fixation location, target number, and eventual estimate. We focused on the first fixation because it is the earliest measure of processing. An LMM predicting first fixation location from number requested found that eye movements were related to the target number even from the first fixation $(\beta=.33, S E=.03, p=.0001)$. Participants' first fixation location was also predictive of their eventual click location $(\beta=.86, S E=.08, p=.0001)$. To explore the relationship between error in first fixation and estimation error, we also calculated differences between the $x$-coordinates of the correct location and (1) a participant's mouse click and (2) the first fixation location, resulting in a negative score for all underestimates and a positive score for all overestimates. An LMM predicting estimation error from error in the first fixation revealed that the amount and direction of error in a participant's first fixation was related to error in their eventual estimate $(\beta=.02, S E=.01, p<.05)$.
Fixations on reference points

Participants' fixation behavior indicated a preference for fixating on the midpoint (see Fig. 1). To examine this pattern statistically, we defined three new regions containing the 40 pixels surrounding three candidate reference points: 250, 500, and 750 on the number line. Because primary analyses indicated that participants spent more time near the correct region, trials requiring estimates within 50 number units of these new regions were removed from analyses (13/40 trials). For the remaining 27 trials, total fixation duration and total number of fixations were subjected to 2 (initial number: small or large) by 3 (region: 250, 500, or 750) ANOVAs. These indicated a main effect of region [duration, $F(2,50)=$ $7.84, M S E=34,350, p<.01$; \# fixations, $F(2,50)=10.71$, $M S E=.113, p<.001]$, but no effect of initial number and no interaction $(p s>.05)$. Participants had longer total fixation durations $(222 \mathrm{~ms})$ in the 500 region, as compared to either the 250 region [65 ms; $t(26)=2.60, p<.025]$ or the 750 region [41 ms; $t(26)=3.15, p<.01]$, which did not differ statistically $(p>.25)$. There were more total fixations on average in the 500 region (.50) than in either the 250 region $[.16 ; t(26)=2.93, p<.01]$ or the 750 region $[.11 ; t(26)=$ $3.62, p<.01]$, which did not differ statistically $(p>.25)$.

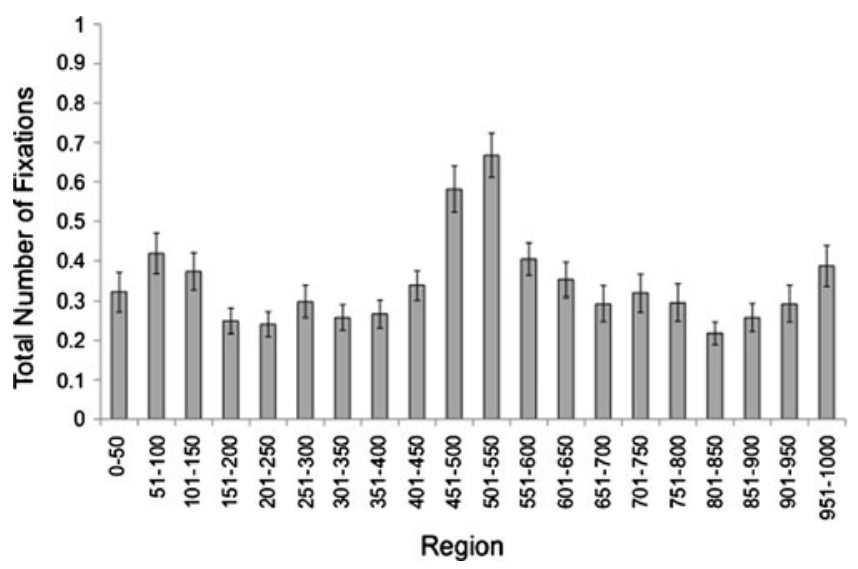

Fig. 1 Mean number of fixations by region. Error bars are SEMs 
Evidence for proportion estimation strategies

The reference point analyses above revealed an explicit tendency to fixate on the line's midpoint. This predicts a specific pattern of bias in participants' estimates (see Barth \& Paladino, 2011; Hollands \& Dyre, 2000). Often, smaller proportions of a whole tend to be overestimated and larger proportions tend to be underestimated (see Spence, 1990; the reverse pattern can also occur, see Cohen \& BlancGoldhammer, in press). This pattern of estimation bias appears between any two reference points (Hollands \& Dyre, 2000). For participants using three reference points (the line's two endpoints plus the midpoint), these models predict overestimation for numbers under 250, underestimation from 250 to 500 , overestimation from 500 to 750 , and underestimation from 750 to 1,000 , with the least error at 0,500 , and $1,000^{2}$ (for further details of these models, see Barth \& Paladino, 2011; Hollands \& Dyre, 2000).

To assess this prediction, we tested three models of estimation bias predicted by reference point use: two reference points ( 0 and 1,000), three reference points $(0,500$, and $1,000)$, and five reference points $(0,250,500,750$, and 1,000). For each model, the target was coded with a number between -1 (extreme underestimation) and 1 (extreme overestimation) based on the degree and direction of error predicted by proportion estimation models for participants using those reference points. We used these values to predict participants' response error for their estimate (estimate location - target location). These analyses again took into consideration all fixations on the number line. In an LMM containing predictors from all three models of response error, only the three-reference-point model $(0,500$, and $1,000)$ significantly predicted the amount and direction of error (two reference points, $\beta=-5.8, S E=4.2, p>.1$; three reference points, $\beta=18.3, S E=4.4, p<.0001$; five reference points, $\beta=2.4, S E=4.4, p>.5$ ). We conducted an additional analysis predicting participants' estimation errors from the three-reference-point model, with Initial Number as a factor. Both initial number and the threereference-point model were significant predictors of the

\footnotetext{
${ }^{2}$ Although the formal three-reference-point model crosses $y=x$ at 250 and 750 , the model does not necessarily predict less error at those points. This is because these intersections are somewhat "accidental" (that is, these intersections do not reflect any particular knowledge of that region of the number line on the part of the observer; rather, they result from the way in which multiple power functions combine to create the proportion judgment model; see Hollands \& Dyre, 2000). Group average data may well reflect lower levels of error at these intersections, but individual data should not necessarily do so (this is especially likely to be true of developmental data). It is only at the actual reference points (in this example, 0,500 , and 1,000) that the model clearly predicts lower levels of error, despite the fact that the formal models do intersect with $y=x$ between the reference points as well.
}

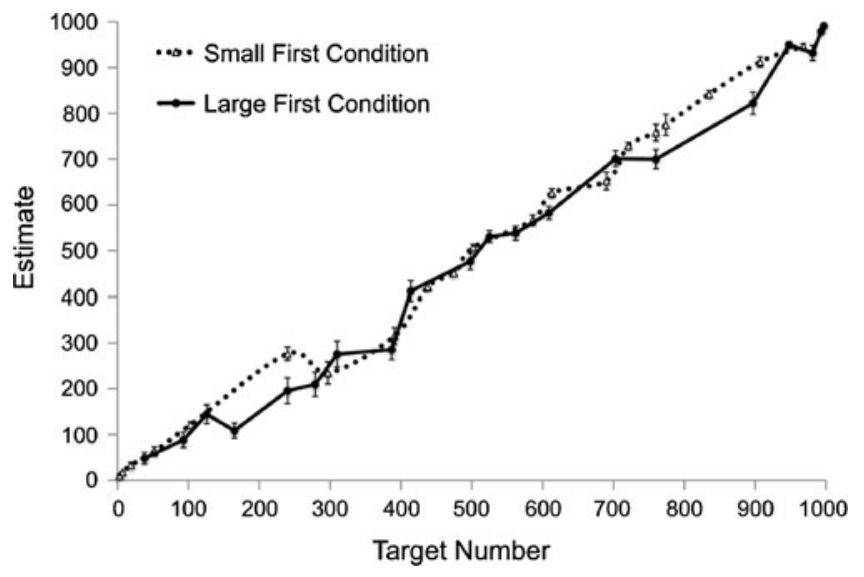

Fig. 2 Estimation behavior by initial-number condition. Error bars are SEMs

amount and direction of error, but there was no interaction (initial number, $\beta=-22.95, S E=7.69, p<.01$; three reference points, $\beta=17.3, S E=5.79, p<.01$; interaction, $p>.5)$. Although small-initial-number participants made larger estimates than did large-initial-number participants, all participants' estimates revealed error patterns consistent with proportional-estimation strategies, and estimation bias was consistent with the use of a central reference point, as predicted by preferential fixation on the midpoint. Estimation performance as a function of initial number and target number is displayed in Fig. 2.

\section{Discussion}

The present study had three main findings. First, adults' translation of numerical information into spatial extent during number-line estimation is rapid and precise: Participants preferentially fixated on and near the correct target location, devoting nearly $50 \%$ of their fixation time to less than $15 \%$ of the number line. The location of the very first fixation on the line was influenced by the target number and was predictive of the participant's eventual estimate. Additionally, the distance of the first fixation from the actual target location was related to estimation error. These findings suggest that estimation error arises early in task processing.

Second, despite this evidence of rapid number/space translation, we found evidence of online calibration of estimates. Participants who initially encountered a relatively small number differed from participants who initially encountered a large number, both in looking behavior and in estimation performance. This finding suggests that the estimation process is dynamic, consistent with previous studies in which participants adjusted estimation strategies to incorporate information about numbers to be estimated (Izard \& Dehaene, 2008; Sullivan \& Barner, 2010). 
Calibration effects can therefore emerge even without explicitly misleading feedback, and because initial-number condition influenced both estimation and fixation behavior, our data suggest that the calibration effects found in previous studies could arise early in processing.

Third, this study found evidence that adults' number-line estimates demonstrate patterns of error predicted by psychophysical models of proportion estimation (Hollands \& Dyre, 2000; Spence, 1990), consistent with recent findings in adults (Cohen \& Blanc-Goldhammer, in press) and children (Barth \& Paladino, 2011). The proportionalreasoning model used in the present article made specific predictions about patterns of estimation bias (over- and underestimation) that should arise in number-line estimation tasks (even for highly accurate adults). These patterns, appearing in our data as predicted by the model, were not predicted by the proportional-reasoning strategy mentioned by Siegler and Opfer (2003), which was based on the hypothesis that landmark use would reduce the variability of estimates around landmarks (making no predictions about the direction of the errors). The proportionalreasoning model discussed here therefore makes specific and accurate predictions about both the number and direction of errors. It is also based on a substantially different theoretical foundation (see Hollands \& Dyre, 2000) than were previous proportional-reasoning accounts (Siegler \& Opfer, 2003), and it provides evidence for a different story about the development of numerical estimation (see Barth \& Paladino, 2011). In both our explicit (behavioral) and implicit (eye movement) measures of estimation performance, adults' estimation behavior showed evidence that their estimates are biased by a proportional-reasoning strategy. Our findings further extend those of Cohen and Blanc-Goldhammer by showing that the specific bias patterns in participants' estimation data are directly predicted by their preferential fixations on the midpoint of the line.

Adults' high levels of accuracy in number-line estimation are undoubtedly supported by their mature understanding of the symbolic number system (see, e.g., Siegler \& Opfer, 2003). Patterns of bias predicted by proportional-reasoning accounts of number-line estimation, however, are present even in the very accurate numerical estimates of adults, and are predicted by participants' fixations during the task. Future research using eye movement measures in children may also prove useful in investigating the developmental course of these abilities. In sum, the present study provides direct evidence, based on estimates and eye movement data, that adults translate numerical information into spatial representations rapidly and in response to subtle differences in task demands, and that they treat number-line estimation tasks as instances of proportion judgment.
Author Note This work was supported by a Jacobs Graduate Fellowship and an NSF Graduate Fellowship to J.S., and by NSF Grant DRL-0950252 to H.B. We thank Keith Rayner for his input on this project, as well as Sarah Creem-Regehr and two anonymous reviewers for their helpful comments.

Open Access This article is distributed under the terms of the Creative Commons Attribution Noncommercial License which permits any noncommercial use, distribution, and reproduction in any medium, provided the original author(s) and source are credited.

\section{References}

Baayen, R. H. (2008). Analyzing linguistic data: A practical introduction to statistics. Cambridge: Cambridge University Press.

Barth, H., \& Paladino, A. (2011). The development of numerical estimation: evidence against a representational shift. Developmental Science, 24, 248-264.

Bates, D., \& Sarkar, D. (2007). Lme4: Linear mixed-effects models using S4 classes ( $\mathrm{R}$ package version 0.9975-12) [Computer software]. Available at http://CRAN.R-project.org/

Booth, J., \& Siegler, R. (2006). Developmental and individual differences in pure numerical estimation. Developmental Psychology, 41, 189-201.

Cantlon, J. F., Cordes, S., Libertus, M. E., \& Brannon, E. M. (2009). Comment on "Log or linear? Distinct intuitions of the number scale in Western and Amazonian indigene cultures". Science, $323,38 \mathrm{~b}$.

Cohen, D. J., \& Blanc-Goldhammer, D. (in press). Numerical bias in bounded and unbounded number line tasks. Psychonomic Bulletin \& Review. doi:10.3758/s13423-011-0059-z

Cohen, D., Ferrell, J., \& Johnson, N. (2002). What very small numbers mean. Journal of Experimental Psychology: General, $131,424-442$.

Dehaene, S., Izard, V., Pica, P., \& Spelke, E. S. (2009). Response to Comment on "Log or linear? Distinct intuitions of the number scale in Western and Amazonian indigene cultures". Science, $323,38 \mathrm{c}$.

de Hevia, M. D., \& Spelke, E. S. (2010). Number-space mapping in human infants. Psychological Science, 21, 653-660.

Ebersbach, M., Luwel, K., Frick, A., Onghena, P., \& Verschaffel, L. (2008). The relationship between the shape of the mental number-line and familiarity with numbers in 5- to 9-year old children: Evidence for a segmented linear model. Journal of Experimental Child Psychology, 99, 1-17.

Heine, A., Thaler, V., Tamm, S., Hawelka, S., Schneider, M., Torbeyns, J., et al. (2010). What the eyes already "know": using eye movement measurement to tap into children's implicit numerical magnitude representations. Infant and Child Development, 19, 175-186.

Hollands, J., \& Dyre, B. (2000). Bias in proportion judgments: the cyclical power model. Psychological Review, 107, 500-524.

Hollands, J., Tanaka, T., \& Dyre, B. (2002). Understanding bias in proportion production. Journal of Experimental Psychology: Human Perception and Performance, 28, 563-574.

Hubbard, E., Piazza, M., Pinel, P., \& Dehaene, D. (2005). Interactions between number and space in parietal cortex. Nature Reviews Neuroscience, 6, 435-448.

Izard, V., \& Dehaene, S. (2008). Calibrating the number line. Cognition, 106, 1221-1247.

Loetscher, T., Bockisch, C., Nicholls, M., \& Brugger, P. (2010). Eye position predicts what number you have in mind. Current Biology, 20, R264-R265. 
R Development Core Team. (2010). $R$ : A language and environment for statistical computing [Computer software]. Vienna, Austria: R Foundation for Statistical Computing.

Schneider, M., Heine, A., Thaler, V., Torbeyns, J., De Smedt, B., Verschaffel, L., et al. (2008). A validation of eye movements as a measure of elementary school children's developing number sense. Cognitive Development, 23, 409-422.

Schwarz, W., \& Keus, I. (2004). Moving the eyes along the mental number line: comparing SNARC effects with saccadic and manual responses. Perception \& Psychophysics, 66, 651664

Siegler, R., \& Opfer, J. (2003). The development of numerical estimation: evidence for multiple representations of numerical quantity. Psychological Science, 14, 237-243.
Siegler, R., \& Ramani, G. (2009). Playing linear number board games — but not circular ones - improves low-income preschoolers' numerical understanding. Journal of Educational Psychology, 101, 545-560.

Siegler, R., Thompson, C., \& Opfer, J. (2009). The logarithmic-tolinear shift: one learning sequence, many tasks, many time scales. Mind, Brain, and Education, 3, 143-150.

Spence, I. (1990). Visual psychophysics of simple graphical elements. Journal of Experimental Psychology: Human Perception and Performance, 16, 683-692.

Sullivan, J., \& Barner, D. (2010). Mapping number words to approximate magnitudes: Associative learning or structure mapping? In S. Ohlsson \& R. Catrambone (Eds.), Cognition in flux: Proceedings of the 32nd Annual Meeting of the Cognitive Science Society (pp. 1246-1251). Austin, TX: Cognitive Science Society. 